\title{
The effects of botulinum toxin type $A$ on improvement and dynamic spastic equinus correction in children with cerebral palsy - preliminary results
}

Hristina Colovic ${ }^{1}$, Lidija Dimitrijevic ${ }^{1,2}$, Ivona Stankovic ${ }^{1,2}$, Dejan Nikolic ${ }^{3}$, Dragana Radovic-Janosevic ${ }^{2,4}$, Dragoljub Zivanovic ${ }^{2,5}$

\author{
${ }^{1}$ Clinic for Physical Therapy and Rehabilitation, Clinical Centre of Nis, Nis, Serbia \\ ${ }^{2}$ Faculty of Medicine, University of Nis, Nis, Serbia \\ ${ }^{3}$ Physical Medicine and Rehabilitation, University Children's Hospital, Belgrade, Serbia \\ ${ }^{4}$ Gynecology and Obstetrics Clinic, Clinical Centre of Nis, Nis, Serbia \\ ${ }^{5}$ Pediatric Surgery Clinic, Clinical Centre of Nis, Nis, Serbia
}

Submitted: 21 February 2012

Accepted: 8 July 2012

Arch Med Sci 2014; 10, 5: 979-984

DOI: 10.5114 /aoms.2014.46217

Copyright (C 2014 Termedia \& Banach

\section{Abstract}

Introduction: We evaluated the effects of botulinum toxin type A (BTA) with physical therapy on dynamic foot equinus correction and higher motor functional outcome in children with spastic type of cerebral palsy (CP).

Material and methods: Ankle joint active and passive movement, gastrocnemial muscle spasticity levels (Modified Ashworth Scale (MAS)), and higher motor functional status (Gross Motor Function Classification System (GMFCS) and Gross Motor Function Measure (GMFM) (GMFM-D - standing and GMFM-E - walking) were assessed before treatment and 3, 8, 16 weeks and 6 months after BTA administration in 12 children.

Results: There was a significant improvement of active (initial - (-) 13.07 $\pm 5.78 ; 6$ months $-(-) 10.64 \pm 4.77 ; p<0.001$ ) and passive (initial -4.21 $\pm 2.29 ; 6$ months $-4.71 \pm 2.16 ; p<0.05)$ ankle joint foot dorsiflexion. GMFM-D and GMFM-E were significantly higher after $3,8,16$ weeks $(p<0.001)$ and GMFM-D after 6 months $(p<0.001)$

Conclusions: Botulinum toxin type $A$ administration and physical therapy in patients with spastic CP improves the motion range of dynamic foot equinus after 3 weeks and higher motor functional outcome (standing and walking).

Key words: cerebral palsy, botulinum toxin, dynamic equines.

\section{Introduction}

Cerebral palsy (CP) refers to a group of non-progressive nervous system disorders with musculoskeletal manifestation due to an early brain developmental disorder [1]. Cerebral palsy may be followed by sensory, affective, perceptive and cognitive impairments with seizure disorders in certain cases. It is assumed that CP is the most frequent cause of physical handicap in childhood, with incidence of 2-2.5 per 1000 newborns [2]. Spasticity is the most frequent neurological presentation of CP, which is manifested mainly on lower extremities and correlates closely with prematurity $[3,4]$. The permanent musculoskeletal pathology in spastic type of CP is acquired during childhood. Several authors have pointed out that a reduction by at least $50 \%$ in the longitudinal growth
Corresponding author: Hristina Colovic MD, PhD Clinic for Physical Therapy and Rehabilitation Clinical Centre of Nis Branka Krsmanovica 17/34 18000 Nis, Serbia Phone: +381641264218 E-mail: ninacol40@ymail.com 
of myofibrils could be associated with the onset of contractures and permanent skeletal-joint system deformities [5-7].

The majority of children with spastic type of CP present with both spasm and contractures, since contractures develop in early stages. The transition time from dynamic to fixed contracture in these patients varies and depends on the type of selective muscle groups. Fixed contracture in unilateral spastic type of CP develops earlier on lower than on upper extremities, pointing to the fact that at the time of surgical correction of rigid equinovarus deformity, there is still a possibility of a successful conservative treatment outcome for the spasticity of upper extremities [8].

It is important to include children with spastic type of CP in early treatment to prevent possible rigid contractures, deformities and correction of acquired improper motor functions. Previous reports advise postponing surgical intervention of spastic equinus until the age of 6 years if possible, since at younger age there is a greater chance for recurrent intervention [9]. The treatment of dynamic contractures and spasticity in CP patients includes administration of botulinum toxin type A (BTA) as well as physical therapy and proper orthoses application. The BTA effects are permanent and include irreversible denervation in the applied region, leading to muscle weakness. In clinical terms, spasticity reduction lasts for 12-16 weeks in the majority of patients, while functional effects can last 6 months and above, followed by the formation of new synapses $[10,11]$.

Previously we have pointed out that there is a positive correlation between BTA treatment and physical therapy, with a reduction in spasticity and overall functional outcome for children with CP [12]. We have postulated that reduction in ankle joint motion spasticity in children with CP who were included in BTA treatment could be in correlation with increase in movement range followed by the increase in angle motion and improvement in higher motor functions (standing and walking).

Therefore, in this study we aimed to evaluate the effects of BTA administration along with physical therapy on correction of dynamic foot equinus (degrees of motion in ankle joint) and higher motor functional outcome (standing and walking) in children with spastic type CP.

\section{Material and methods}

\section{Study group}

This prospective study included 12 children age between 2 to 6 years with both unilateral and bilateral spastic type of CP. The abobotulinumtoxi$n A(A B O), B T A-A B O$, was applied into the gastrocnemial muscle for the purpose of dynamic foot equinus correction. Before inclusion in the study parents or legal guardians were informed about the treatment protocol and informed consent was obtained. The study was approved by the Institutional Review Board of the Faculty of Medicine and carried out according to the principles of good clinical practice. The BTA-ABO administration was done by a board certified physiatrist.

Criteria for inclusion in the study were as follows: obtaining informed consent, dynamic contracture of ankle joint with inadequate response (absence of spasticity reduction over 2 months of continuous physical therapy) to the physical treatment, patients able to walk with or without help, patients who attended a re-habilitation program regularly. Exclusion criteria were as follows: rigid contractures of ankle joint, significant difference between length of lower limbs $(>5 \mathrm{~cm})$, significant lower limb muscular atrophy, surgical correction on ankle joint or foot performed, previously applied injection with alcohol medium in gastrocnemial muscle, and irregular attendance in a re-habilitation program.

One ampoule of BTA-ABO that was used in the study consisted of 500 international units (IU) and was dissolved before application in $2.5 \mathrm{ml}$ of sterile $0.9 \% \mathrm{NaCl}$ solution. Total drug dosage was distributed in both gastrocnemial muscle heads (caput laterale et mediale), so in total we treated 16 lower extremities with dynamic spastic foot equinus. Application of BTA-ABO was determined by palpation on 2 or 4 applicable places in the gastrocnemial muscle, according to the length of the distal part of the lower limb below the knee. Total drug dosage was calculated according to the child body weight, so for children with unilateral form it was $10 \mathrm{lU} / \mathrm{kg}$, while for those with bilateral form it was $20 \mathrm{IU} / \mathrm{kg}$. If needed, according to the decision of a board certified physiatrist, diazepam suppositories were given for sedation one hour prior to BTA-ABO application.

Physical therapy was individually designed and included thermotherapy, kinesiotherapy (exercises included movement range increase, ankle dorsiflexion facilitation, muscle stretching, antagonists strengthening, gait training, coordination, and correction of acquired improper motor functions), occupational and functional therapy, and prescription of adequate orthoses for deformity correction. The program was initiated 5 days after BTA-ABO application, with the plan of the standardized physical therapy to be performed 3 times weekly in duration of $1 \mathrm{~h}$ per child [13]. For the purpose of this study the physical therapy program lasted for 16 weeks.

The effects of BTA-ABO application and applied physical therapy were estimated by the analysis of active and passive movement in the ankle joint, gastrocnemial muscle spasticity and 
motor functional status (measured by Gross Motor Function Measurement (GMFM)). These parameters were measured 5 times: before treatment, 3, 8, 16 weeks after and 6 months after the treatment.

Active and passive movement in the ankle joint was measured using a standardized goniometer and was expressed by angle degrees. Active joint movement was evaluated through active cooperation with the patient (the patient performs an active movement on request), while passive ankle movement was performed by the examiner, with the exclusion of compensatory movements. Inability to perform foot dorsiflexion up to the neutral position was indicated with a negative sign $(-)$. The degree of spasticity was estimated by the Modified Ashworth Scale (MAS) with performed passive movement of the ankle in a supine lying position [14].

Motor functional status of children with CP was estimated by GMFM, which is considered as the gold standard and a valid instrument for quantification of how many motor functions the child can perform and is specially designed for CP patients [15]. We applied the GMFM-88 test with 88 motor functions divided into 5 categories: $A$ - lying and rolling (17), B - sitting (20), C - crawling and kneeling (14), D - standing (13), E - walking, running, jumping (24) [15]. The score was expressed as a percentage. The study included presentation of treatment effects on the standing category (GMFM-D) and walking category (GMFM-E), which represents the highest level of acquired motor functions, since the CP patients in this study were able to walk with or without help.

\section{Statistical analysis}

The data were presented as arithmetic mean values ( $x$ ) with standard deviation (SD) and 95\% confidence interval $(95 \% \mathrm{Cl})$. To evaluate statistical difference between mean values at different times of observation and between groups we used Student's t-test for dependent samples. In cases where the distribution of parameters showed deviation from normal we used the Wilcoxon signed ranks test to test the statistical difference between mean values. Pearson's $\chi^{2}$ test was used to evaluate the distribution of categorical variables. Statistical significance was set at $p<0.05$.

\section{Results}

The average age of evaluated participants with spastic type of CP from the study was $4.38 \pm 0.11$ years and average body weight was $19.48 \pm 4.12 \mathrm{~kg}$. The average applicable dose of BTA-ABO per patient was $215.01 \pm 112.04 \mathrm{IU}$, ranging from $100 \mathrm{IU}$ to $480 \mathrm{IU}$.

There was a highly significant difference in mean values of active foot dorsiflexion in the ankle joint after BTA-ABO application versus initial values before treatment $(p<0.001)$ (Table I). The values of foot dorsiflexion after 6 months, even though they were close to the initial values, were still highly significantly different $(p<0.001)$ (Table I). Mean values of passive foot dorsiflexion at 3, 8 and 16 weeks after the beginning of the treatment highly significantly differed (higher values) versus the initial values $(p<0.001)$ (Table II). At 6 months after BTA-ABO application there was still a significant difference $(p<0.05)$ in mean values but the mean value was closer to the initial one (Table II).

When spasticity levels of extensor muscles are compared according to the MAS, it is observed that there is a significantly higher proportion of those patients with lower spasticity levels at 3, 8 and 16 weeks after the beginning of the treatment versus initial values $(p<0.001)$. After 6 months there was non significant change in the proportion of patients regarding spasticity levels, even though a distribution trend of lower spasticity levels was noticed (Table III).

Mean values of GMFM-D score after 3, 8, 16 weeks, as well as after 6 months, statistically significantly differ (higher values) versus the initial value before treatment of BTA-ABO $(p<0.001)$ (Table IV). It was noted that mean values of GMFM-D

Table I. Motion mean values and statistical interpretation of active foot dorsiflexion

\begin{tabular}{|lccccc|}
\hline & \multicolumn{1}{c}{ Period } & $\boldsymbol{N}$ & $\boldsymbol{X} \pm$ SD & Significance & $95 \% \mathrm{Cl}$ \\
\hline $\begin{array}{l}\text { Active foot } \\
\text { dorsiflexion } \\
\text { (degrees) }\end{array}$ & Before treatment & 16 & $(-) 13.07 \pm 5.78$ & & $(-) 16.41-(-) 9.73$ \\
\cline { 2 - 6 } & After BTA-ABO treatment: & & & $1.36-7.22$ \\
\cline { 2 - 6 } & 3 weeks & 16 & $4.29 \pm 5.08$ & $p<0.001^{\text {a }}$ & $8.05-13.67$ \\
\cline { 2 - 6 } & 16 weeks & 16 & $7.71 \pm 4.38$ & $p<0.001^{\text {b }}$ & $5.19-10.24$ \\
\cline { 2 - 6 } & 6 months & 16 & $(-) 10.64 \pm 4.77$ & $p<0.001^{\text {a }}$ & $(-) 13.39-(-) 7.89$ \\
\hline
\end{tabular}

${ }^{a}$ Student's $t$-test, ${ }^{b}$ Wilcoxon signed ranks test, $N$ - number of extremities 
Table II. Motion mean values and statistical interpretation of passive foot dorsiflexion

\begin{tabular}{|c|c|c|c|c|c|}
\hline & Period & $N$ & $X \pm \mathrm{SD}$ & Significance & $95 \% \mathrm{Cl}$ \\
\hline \multirow{6}{*}{$\begin{array}{l}\text { Passive foot } \\
\text { dorsiflexion } \\
\text { (degrees) }\end{array}$} & Before treatment & 16 & $4.21 \pm 2.29$ & & $2.89-5.54$ \\
\hline & \multicolumn{5}{|c|}{ After BTA-ABO treatment: } \\
\hline & 3 weeks & 16 & $14.64 \pm 3.13$ & $p<0.001^{a}$ & $12.84-16.45$ \\
\hline & 8 weeks & 16 & $15.36 \pm 4.18$ & $p<0.001^{b}$ & $12.94-17.77$ \\
\hline & 16 weeks & 16 & $13.07 \pm 4.23$ & $p<0.001^{a}$ & $10.63-15.52$ \\
\hline & 6 months & 16 & $4.71 \pm 2.16$ & $p<0.05$ & $3.47-5.96$ \\
\hline
\end{tabular}

aStudent's t-test, ${ }^{b} W i l c o x o n$ signed ranks test, $N$ - number of extremities

Table III. Comparison of frequency of ankle extensors spasticity level according to MAS

\begin{tabular}{|c|c|c|c|c|c|}
\hline \multirow{2}{*}{$\begin{array}{l}\text { Level } \\
\text { of spasticity }\end{array}$} & \multirow{2}{*}{$\begin{array}{c}\text { Before } \\
\text { treatment }\end{array}$} & \multicolumn{4}{|c|}{ After BTA-ABO treatment } \\
\hline & & 3 weeks & 8 weeks & 16 weeks & 6 months \\
\hline 0 & 0 & 9 & 11 & 1 & 0 \\
\hline 1 & 0 & 5 & 4 & 9 & 0 \\
\hline $1+$ & 1 & 2 & 1 & 5 & 1 \\
\hline 2 & 5 & 0 & 0 & 1 & 9 \\
\hline 3 & 10 & 0 & 0 & 0 & 6 \\
\hline 4 & 0 & 0 & 0 & 0 & 0 \\
\hline Total & 16 & 16 & 16 & 16 & 16 \\
\hline Value of $p$ & & $<0.001^{*}$ & $<0.001^{\star}$ & $<0.001^{*}$ & NS \\
\hline
\end{tabular}

${ }^{\star}$ Pearson $\chi^{2}$ test

Table IV. The GMFM-D scores in evaluated group of patients before and after BTA-ABO application

\begin{tabular}{|c|c|c|c|c|c|}
\hline & Period & $N$ & $X \pm \mathrm{SD}$ & Significance & $95 \% \mathrm{Cl}$ \\
\hline \multirow{6}{*}{$\begin{array}{l}\text { GMFM-D } \\
(\%)\end{array}$} & Before treatment & 12 & $68.64 \pm 14.67$ & & $64.07-73.21$ \\
\hline & \multicolumn{5}{|l|}{ After treatment: } \\
\hline & 3 weeks & 12 & $75.59 \pm 12.76$ & $p<0.001^{*}$ & $71.62-79.57$ \\
\hline & 8 weeks & 12 & $82.64 \pm 12.90$ & $p<0.001^{\star}$ & $78.62-86.65$ \\
\hline & 16 weeks & 12 & $87.25 \pm 11.63$ & $p<0.001^{*}$ & $83.62-90.87$ \\
\hline & 6 months & 12 & $71.59 \pm 15.14$ & $p<0.001^{\star}$ & $66.87-76.31$ \\
\hline
\end{tabular}

${ }^{*}$ Wilcoxon signed ranks test, $N$ - number of subjects

score 6 months after the BTA-ABO treatment are closer to initial values (Table IV). Mean values of GMFM-E score after 3, 8 and 16 weeks statistically significantly differ (higher values) versus initial values before BTA-ABO treatment $(p<0.001)$ (Table V). There is no significant difference in mean values of GMFM-E scores between initial values and 6 months after the beginning of treatment (Table V).

\section{Discussion}

The consensus of BTA use for the treatment of lower extremity spasticity in children with spastic type of CP was based on the analysis of professional opinions and literature from the In- ternational Committee for BTA treatment [3]. The results were published in the form of recommendations in 2010, and it is further pointed out that BTA injection is effective treatment for spasticity in CP children, which should be combined with physical therapy and application of orthoses. It should be stated as well that the results over 20 years of BTA application in children with CP have proven that such treatment has undoubted positive therapeutic effects on reducing spasticity; therefore, methodologically, in our study we did not follow the effects of BTA application compared to the control group.

It is noted that reduction of spasticity influences different functional aims in different groups 
Table V. The GMFM-E scores in evaluated group of patients before and after BTA-ABO application

\begin{tabular}{|lccccc|}
\hline & \multicolumn{1}{c}{ Period } & $\boldsymbol{N}$ & $\boldsymbol{X} \pm$ SD & Significance & $95 \% \mathrm{Cl}$ \\
\hline $\begin{array}{l}\text { GMFM-E } \\
(\%)\end{array}$ & Before treatment & 12 & $55.49 \pm 20.87$ & & $48.99-61.99$ \\
\cline { 2 - 6 } & After treatment: & & & & \\
\cline { 2 - 6 } & 3 weeks & 12 & $62.52 \pm 19.06$ & $p<0.001^{\mathrm{a}}$ & $56.58-68.46$ \\
\cline { 2 - 6 } & 8 weeks & 12 & $70.83 \pm 18.57$ & $p<0.001^{\mathrm{b}}$ & $71.99-82.01$ \\
\cline { 2 - 6 } & 16 weeks & 12 & $77.00 \pm 16.07$ & $51.81-64.49$ \\
\hline
\end{tabular}

${ }^{a}$ Student's t-test, ${ }^{b}$ Wilcoxon signed ranks test, $N$ - number of subjects

of patients, but the common characteristic for all of them is an increase of movement range. We found that there was a highly significant increase in active movement range in the ankle joint for the group of CP children with spastic foot equinus at 3 weeks after BTA-ABO application and the significance was maintained throughout the entire investigation $(p<0.001)$. Such increase in active movement range maintains its positive trend with the peak in the $8^{\text {th }}$ week after the beginning of treatment. In the period between 16 weeks and 6 months after the BTA-ABO treatment there was a decline in the mean values of active movement range, but still with a significant difference versus initial values $(p<0.001)$. These findings correlate closely with reports of previous studies, stating that there is a significant increase in movement range 3 weeks after BTA administration that is maintained throughout the $16^{\text {th }}$ week after BTA application but with slightly lower statistical significance $(p<0.01)[16,17]$. Such a difference in statistical interpretation is due to the study design, since most of the reports base their findings on comparisons between experimental and control groups, while in our study we observed the increase of movement range before and after therapy in a group of children with CP. As for the active foot dorsiflexion, our results showed a highly significant increase in passive movement range in ankle joint dorsiflexion for the group of $\mathrm{CP}$ children with spastic foot equinus at 3 weeks after BTA-ABO application ( $p<0.001)$, which maintained significance throughout the entire investigation, but after 6 months at the level of $p<0.05$ (Table II). It is important to stress that there is significant benefit of even a small increase of active and passive movement range in ankle joint dorsiflexion for standing and walking as well as for prevention of rigid contractures.

Walking dysfunction, which is primarily conditioned by the spasm of affected muscles in lower extremities, presents the dominant characteristic of spastic type of CP. The basis of the therapeutic approach is to identify and treat spastic muscles or muscle groups in order to increase the movement range in affected extremities, prevent contractures and deformities, and to encourage proper muscle and bone growth. It was noted in our study that there was a highly significant decrease in spasticity of the gastrocnemial muscle $(p<0.001)$ at 3 weeks after BTA-ABO application and significance was maintained until 16 weeks after beginning treatment according to the MAS. However, even though there was no significant difference in the values after the period of 16 weeks, it was noted that spasticity levels were lower according to the MAS. Regarding the estimation of gastrocnemial muscle spasm, 10 muscles had grade 3 before BTA-ABO application, while 6 months after the treatment, 4 of them presented with lower spasticity levels. In the work of Wong, spasticity reduction after BTA application was presented as an average decrease by 1.19 for gastrocnemial muscle, 1.12 for adductors and 2.0 for extensors [18]. In our opinion, such an interpretation of spasticity reduction is statistically precise but without qualification. From the results of our study it can be seen that change in motion range (angle degrees) is not in close correlation with the spasticity levels at 6 months after BTA administration. Even though there was a decline in spasticity (MAS) in the evaluated group of patients, we demonstrated significant changes in angle degrees of ankle joint motion range. Such findings suggest that even lower reduction in spasticity in children with spastic type of CP could result in significant changes of motion range of ankle joints, leading to better balance.

It is relatively simple to estimate spasticity reduction and movement range improvement, but the evidence of functional gains is more complex to estimate. It is essential to stress that measures of functions and abilities are of great significance in estimation of BTA treatment effects in children with CP. Recent findings indicate that BTA treatment in children with CP could reduce energy consumption, resulting in functional improvement [19]. Our results showed that BTA administration correlates closely with improvement in functions (standing (GMFM-D) and walking (GMFM-E)) and reduces spasticity. For improvement of walking ability a longer period of time is needed, and a significant component in this process is habilitation treatment. This is explained from the results 
of our study by the fact that mean values of the GMFM score were higher for standing (GMFM-D) and lower for walking (GMFM-E). Several studies have reported the possible influence of BTA on improvement in walking from short term (up to 3 months after the treatment) $[20,21]$ to long term (2 years and above) [22]. Gains in GMFM-E, 6 months after the beginning of the treatment suggest how important habilitation is, aside BTA administration. One possible explanation for the non-significant improvement in GMFM-E at 6 months after BTA-ABO application and physical treatment could be the fact that there was no significant reduction in spasticity levels that prevent more stable coordination and proprioception during the motion of the body (walking).

In conclusion, BTA administration along with physical therapy in patients with spastic type of $C P$ improves the motion range of dynamic foot equinus in the ankle joint after 3 weeks following initial application as well the higher motor tasks (standing and walking). Continuous and individually assessed physical therapy with duration not shorter than 16 weeks is needed for patients with CP.

\section{References}

1. Bhardwaj P, Sabapathy SR. Assessment of the hand in cerebral palsy. Indian J Plast Surg 2011; 44: 348-56.

2. Stanley F, Blair E, Alberman E. Cerebral palsies: epidemiology and causal pathways. MacKeith Press, London 2000.

3. Love SC, Novak I, Kentish M, et al. Botulin toxin assessment, intervention and after-care for lower limb spasticity in children with cerebral palsy: international consensus statement. Eur J Neurol 2010; 17 (Suppl. 2): 9-37.

4. Camargo $\mathrm{CH}$, Teive HA, Zonta $\mathrm{M}$, et al. Botulinum toxin type $A$ in the treatments of lower-limb spasticity in children with cerebral palsy. Arq Neuropsiquiatr 2009; 67: 62-8.

5. Zwick EB, Leistritz L, Milleit BVS, Zwick G, Galicki M, Witte HGS. Classification of equinus in ambulatory children with cerebral palsy - discrimination between dynamic tightness and fixed contractures. Gait Posture 2004; 20: 273-9.

6. Graham HK. Absence of reference to progressive musculoskeletal pathology in definition of cerebral palsy. Dev Med Child Neurol 2006; 48: 78-9.

7. Van der Krogt MM, Doorenbosch CA, Becher JG, Harlaar J. Dynamic spasticity of plantar flexor muscles in cerebral palsy gait. J Rehabil Med 2010; 42: 656-63.

8. Barnes $M$, Johnson $G$. Upper motor neurone syndrome and spasticity. 2th ed. Cambridge University Press, Cambridge 2008 .

9. Seyler TM, Smith BP, Marker DR, et al. Botulinum neurotoxin as a therapeutic modality in orthopaedic surgery: more than twenty years of experience. J Bone Joint Surg Am 2008; 90 (Suppl. 4): 133-45.

10. Jefferson RJ. Botulinum toxin in the management of cerebral palsy. Dev Med Child Neurol 2004; 46: 491-9.

11. Patel DR, Soyode O. Pharmacologic intervention for reducing spasticity in cerebral palsy. Indian J Pediatr 2005; 72: 869-72.
12. Colovic H, Dimitrijevic L, Stankovic I, Nikolic D, Radovic Janosevic D. Estimation of botulinum toxin type A efficacy on spasticity and functional outcome in children with spastic cerebral palsy. Biomed Pap Med Fac Univ Palacky Olomouc Czech Repub 2012; 156: 41-7.

13. Ibrahim Al, Hawamdeh ZM, Al-Qudah AA. Functional outcome of botulinum toxin injection of gastrocnemius and adductors in spastic hemiplegic cerebral palsied children. Eura Medicophys 2007; 43: 13-20.

14. Assessment of spasticity, part I in: Cornerstone therapy education Accessed on line October 5, 2005. Available from:http://www.cornerstoneceu.com/spasticityassessment.htm

15. Russell DJ, Rosenbaum PJ, Avery LM, Lane M. Gross motor function measure (GMFM-66 and GMFM-88) user's manual. Mac Keith Press, London, United Kingdom 2007.

16. Dimitrijević L, Stanković I, Zivković V, Mikov A, Colović H, Janković I. Botulinum toxin type A for the treatment of spasticity in children with cerebral palsy. Vojnosanit Pregl 2007; 64: 513-8.

17. Hayek S, Gershon A, Wientroub S, Yizhar Z. The effect of injections of botulinum toxin type A combined with casting on the equinus gait of children with cerebral palsy. J Bone Joint Surg Br 2010; 92: 1152-9.

18. Wong V. Use of botulinum toxin injection in 17 children with spastic cerebral palsy. Pediatr Neurol 1998; 18: 124-30

19. Balaban B, Tok F, Tan AK, Matthews DJ. Botulinum toxin a treatment in children with cerebral palsy: its effects on walking and energy expenditure. Am J Phys Med Rehabil 2012; 91: 53-64.

20. Koman LA, Mooney JF 3rd, Smith BP, Walker F, Leon JM Botulinum toxin type A neuromuscular blockade in the treatment of lower extremity spasticity in cerebral palsy: a randomized, double-blinded, placebo-controlled trial. BOTOX Study Group. J Pediatr Orthop 2000; 20: 108-15.

21. Papadonikolakis AS, Vekris MD, Korompilias AV, Kostas JP, Ristanis SE, Soucacos PN. Botulinum A toxin for treatment of lower limb spasticity in cerebral palsy: gait analysis in 49 patients. Acta Orthop Scand 2003; 74: 749-55.

22. Cosgrove AP, Corry IS, Graham HK. Botulinum toxin in the management of the lower limb in cerebral palsy. Dev Med Child Neurol 1994; 36: 386-96. 